\title{
Presurgical Nasoalveolar Molding in a Neonate with Unilateral Cleft Lip and Palate
}

\author{
${ }^{1}$ Arun Kumar, ${ }^{2}$ Saumil Mogre
}

\begin{abstract}
The innate fissure of lip and hard palate represents the most common inborn craniofacial oddity originated by unusual facial development through gestation. It varies in form and severity. The rehabilitation amounts to a committed approach of care for the afflicted child since its detection. The craniofacial team works together to arrange the complex surgical and nonsurgical treatment plan. The diverse stratagem has been endeavored to lessen the cleft space and achieve esthetic results postsurgically. The presurgical nasoalveolar molding (PNAM) technique represents an essential part of the treatment of child with orofacial split. It ameliorates the orientation of the palatal ridges and decreases the distance between the cleft lip segments, provides symmetry to severely disfigure nasal gristle. The case report presents a concise insight concerning PNAM approach in unilateral cleft lip and palate (CLCP) patient with excellent presurgical outcome.
\end{abstract}

Keywords: Cleft lip, Cleft palate, Congenital, Presurgical nasoalveolar molding, Unilateral.

How to cite this article: Kumar A, Mogre S. Presurgical Nasoalveolar Molding in a Neonate with Unilateral Cleft Lip and Palate. J Oral Health Comm Dent 2018;12(2):67-72.

Source of support: Nil

Conflict of interest: None

\section{INTRODUCTION}

The orofacial cleft includes inborn fissure of lip, cleft palate $(\mathrm{CP})$, and both together. They are grouped as rare type of congenital deformations. The resulted alterations lead to difficult feeding, articulation issue, listening sound problems, and commonly ear contagion. The surgical approach is a definitive management strategy. This is often within first few months for cleft lip and before 18 months for fissured hard palate. The CLCP deformities are complex with the involvement of not only the palate and lip but also the nose. The malformation represents

\footnotetext{
${ }^{1}$ Associate Professor, ${ }^{2}$ Postgraduate Student

1,2Department of Pedodontics and Preventive Dentistry, Post Graduate Institute of Dental Sciences, Rohtak, Haryana, India

Corresponding Author: Arun Kumar, Associate Professor Department of Pedodontics and Preventive Dentistry, Post Graduate Institute of Dental Sciences, Rohtak, Haryana, India Phone: +919896200192, e-mail: drarun922@gmail.com
}

as disfigurement, displacement, and absence matter of nose and maxillary structures. ${ }^{1}$

The worldwide view depicts 1:600 incidences for $\mathrm{CP}$ children. The rate is most prevalent within Asians populations trailed by Caucasians and Africans. The annual prediction rate of CLCP in India is over 35,000 children. $^{2}$

The achievement of favorable esthetic poses notable surgical problem in patients with cleft element. The varied surgical treatment options have been tried in these patients so as to achieve acceptable outcome. The authors illustrated a wide variety of individualized surgical treatment approaches for resolution of CLCP. ${ }^{3,4}$ Furthermore, to improvise the favorable outcome of lip repair, the rationale of PNAM was evolved. ${ }^{5}$

Presurgical nasoalveolar molding in infants plays a vital part in neonatal CLCP treatment. ${ }^{6}$ McNeil ${ }^{7}$ stated the traditional view of presurgical orthopedics in 1950 wherein the approximation of alveolar cleft segment was done using serial appliances. The present-day nasoalveolar molding works on the same principle. Moreover, the nasoalveolar molding encourages immature nasal chondroblasts, resulting in interstitial tissue expansion and esthetic in the nasal morphology. ${ }^{8}$

The PNAM can be an accompaniment to ease surgical improvement in newborn with CLCP. The principal focus of PNAM is a resolution of soft tissue as well as cartilaginous distortion and helps toward surgical repair with resultant minimizing of scar generation. ${ }^{9,10}$ The PNAM therapy is suitable for tongue position, encourages in proper pronunciation, enhances esthetic and psychosocial parameters with refined feeding and skeletal curvature. ${ }^{6}$ The PNAM mechanism includes removable maxillary alveolar molding appliance. The nasal stent is an integral part of the appliance and is usually incorporated over front portion of the molding plate. It is made up of orthodontic stainless steel wire of 19 gauges. The intraoral PNAM appliance and the nasal stent are adjusted preferably on a weekly basis and gradually correct the deformation. The PNAM is indicated in a complete scale of cleft deformation consisting of unilateral or bilateral orofacial malformation without an unimpaired nasal floor. ${ }^{9}$ The author showed here a case with astonishing result of well-timed PNAM therapy in a unilateral CLCP patient. 


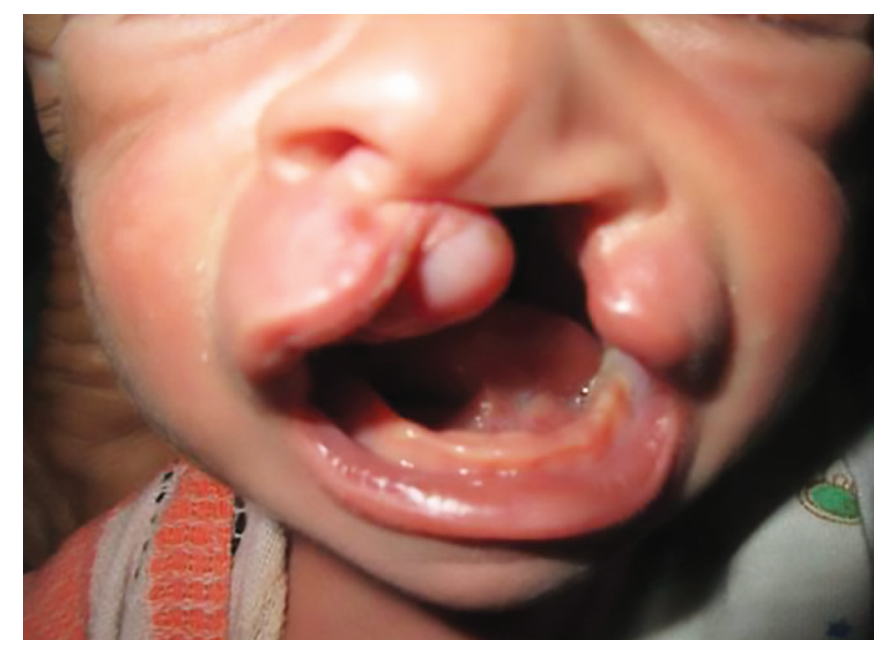

Fig. 1: Preoperative extraoral view

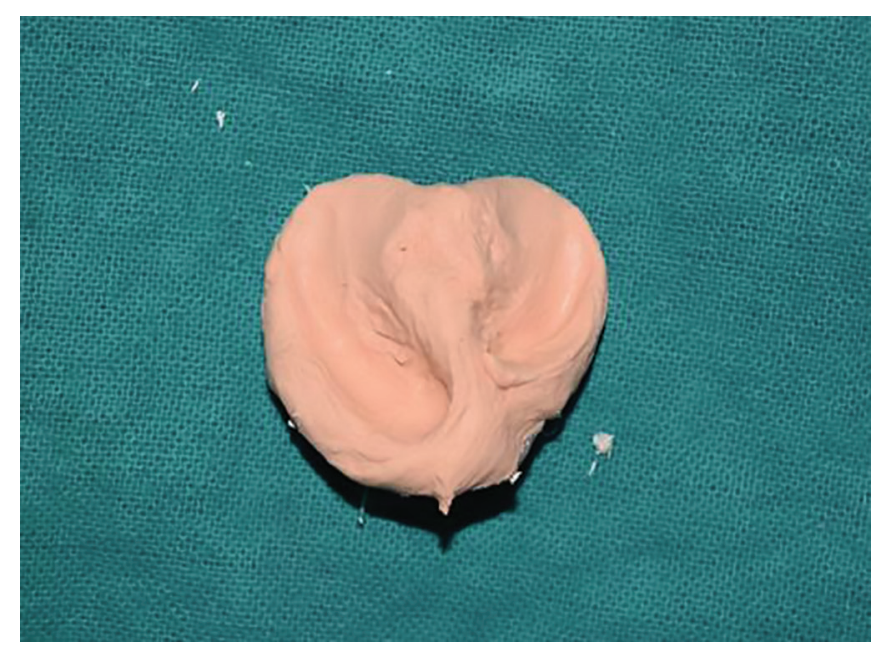

Fig. 3: The initial impression taken using alginate impression material

\section{CASE REPORT}

A 15-day-old baby boy with unilateral CLCP was referred to the Department of Pedodontics and Preventive Dentistry from Plastic Surgery Department for presurgical orthopedic procedure. The previous medical background revealed uneventful pregnancy of mother with no other significant history. The extraoral evaluation revealed total unilateral CLCP of left portion involving the upper lip, nasal alae, and the alveolus. Furthermore, the left nasal rim was collapsed and nasal septum was deviated toward the right side (Fig. 1). The intraoral inspection depicts complete clefting encompassing premaxillary alveolus, hard palate, and soft palate toward the left side (Fig. 2). The CLCP was classified as Veau's class III.

The patient was placed for active PNAM therapy. The extraoral measurement including columella width, columella length, and interlip distance was recorded using a ruler. For intraoral measurements and assembly of orthopedic moldable device for nasoalveolar molding, an impression was planned. The initial impression was

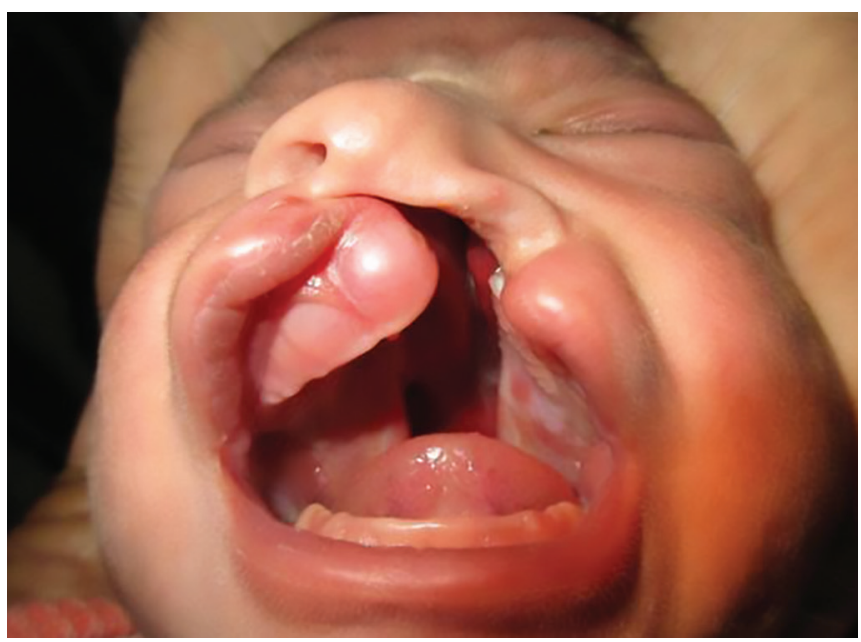

Fig. 2: Preoperative intraoral view

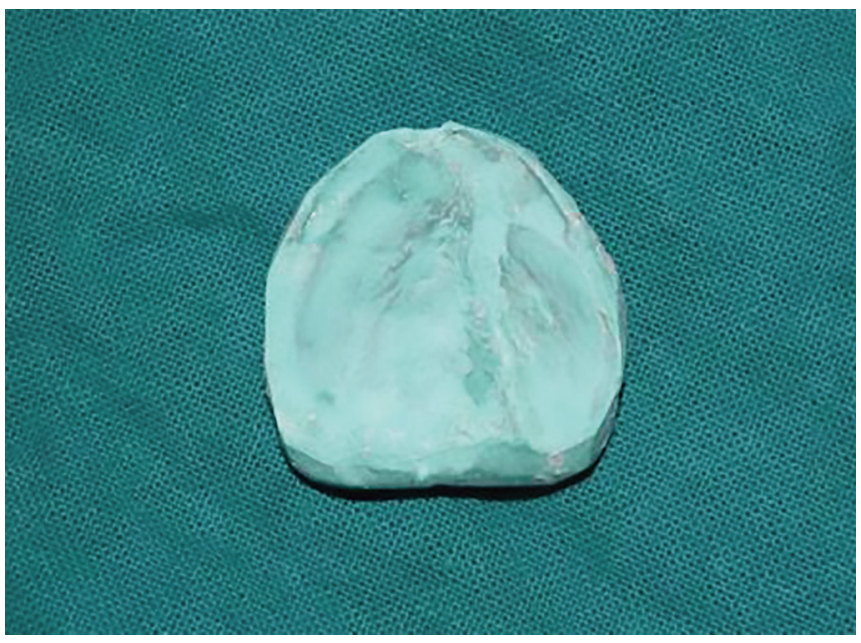

Fig. 4: The primary cast

taken using alginate impression material over a primary impression tray made up of modeling wax (Fig. 3). Just before impression, the intraoral cleft space was blocked using gauge piece with one end secured by tying a suture thread. Another end of braided silk suture was placed extraorally. The primary cast was poured (Fig. 4) and a customized impression acrylic tray was fabricated. The sharp edges of the tray were rounded using sandpaper. The final impression was taken with putty silicon material (Fig. 5) while maintaining child in an erect posture and blocking the cleft space with gauze piece in a manner similar to that for primary impression. A cast was customized and trimmed approximately. All the intraoral measurements (intersegmental distance, arch width, greater segment length, and lesser segment length) were measured with ruler and finally recorded and documented for further reference. All the undercuts were blocked followed by application of separating media/vaseline to the prepared cast. The self-cure acrylic resin was used with sprinkleon technique to create the molding appliance (Fig. 6). 


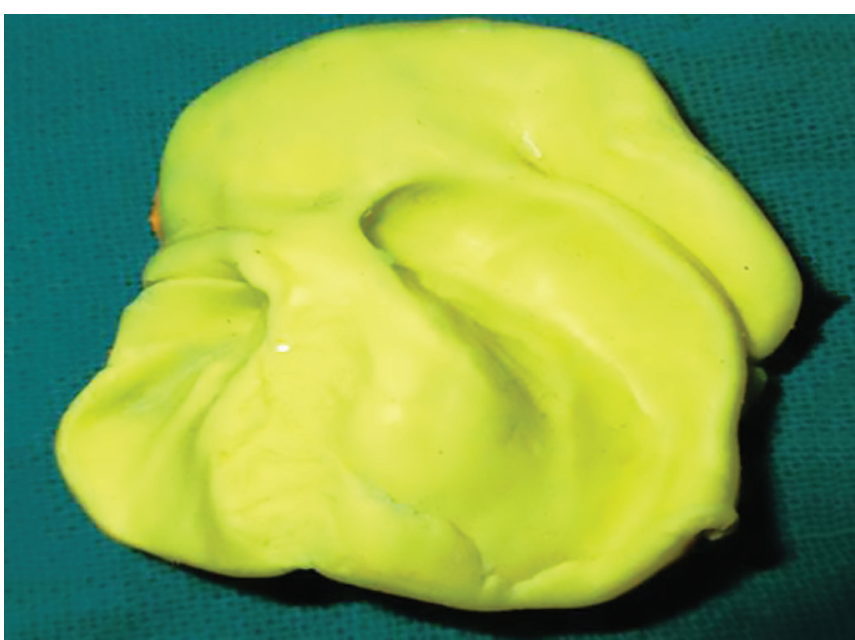

Fig. 5: Final impression with putty silicon material

The nasal extension in the form of stent was made and activated along with PNAM component on the 1st day. The nasal stent was made up of stainless steel and was linked to the PNAM appliance. The end of the nasal stent was made up of acrylic and was positioned along the medial wall of the flawed nares. An acrylic handle was fabricated along with a rim of depression for attachment of blue elastics. After insertion, the appliance was stabilized using Steri-Strips adhesive tape. Unfortunately, the desired adhesiveness was not obtained using Steri-Strips. Thereafter, micropore tapes were used for stabilization of the appliance in position. The micropore tape attached alongside with blue elastics was extended up to limits to apply appropriate force desired for orthopedic maneuver (Fig. 7). The blue elastics and the micropore tape were changed at optimal interval for keeping adequate pressure. The appliance was delivered and put under parent's supervision for first 24 hours. The parents were instructed to separate it solely for cleaning purposes. The patient was recalled for regular weekly follow-up visits. The intraoral alveolar fissure was measured with ruler to evaluate the closure, along with requisite adaptions to the molding device. These were done by shedding the hard acrylic in the zone of advancement (buccal flange and

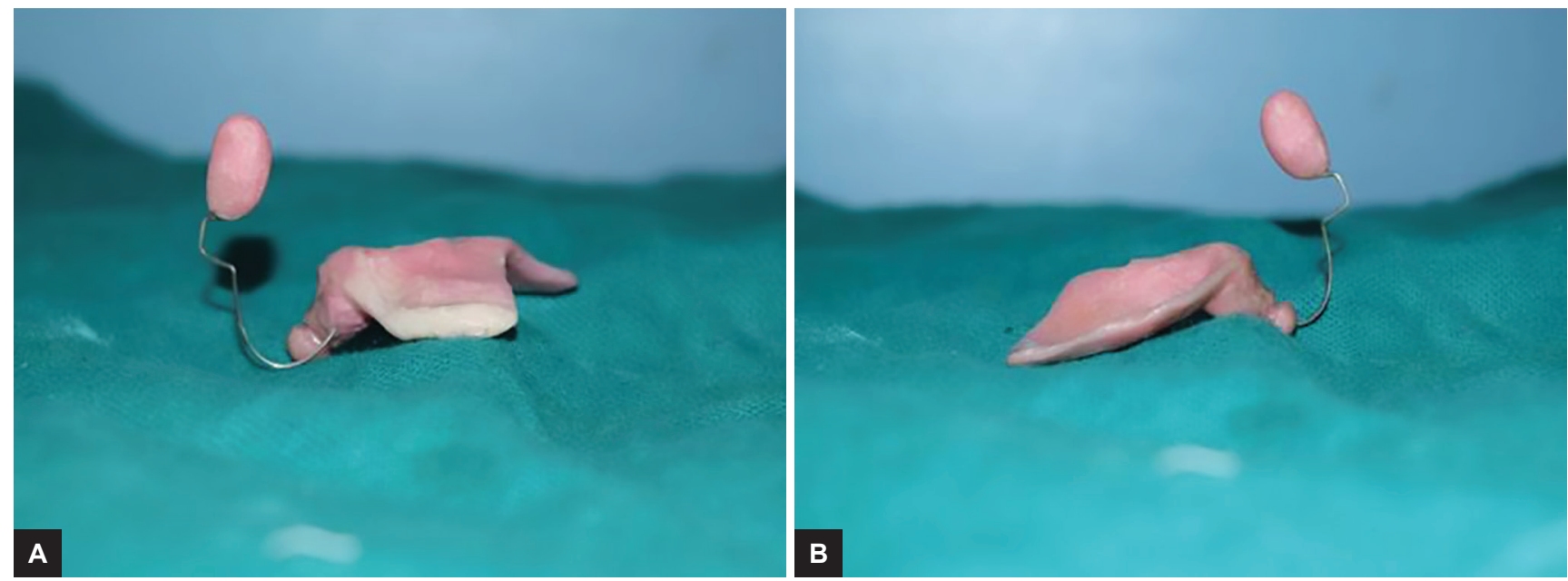

Figs 6A and B: Nasoalveolar molding appliance with nasal stent
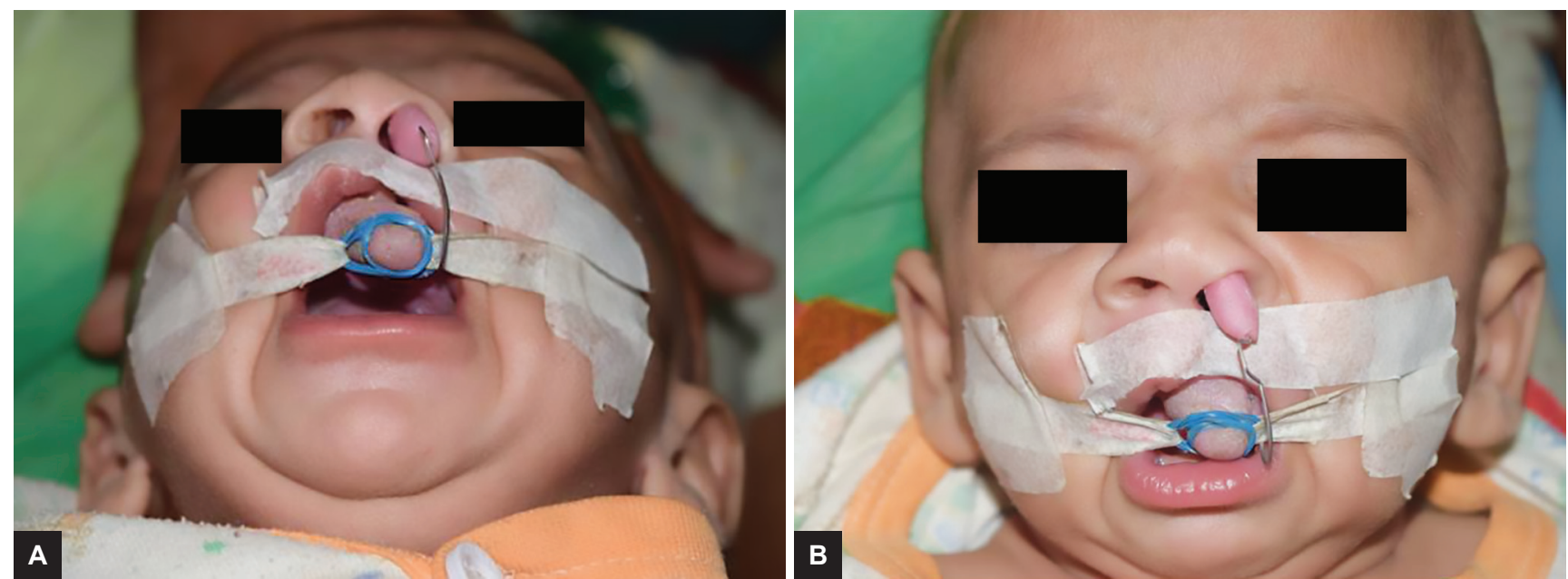

Figs 7A and B: Presurgical nasoalveolar molding appliance in situ 

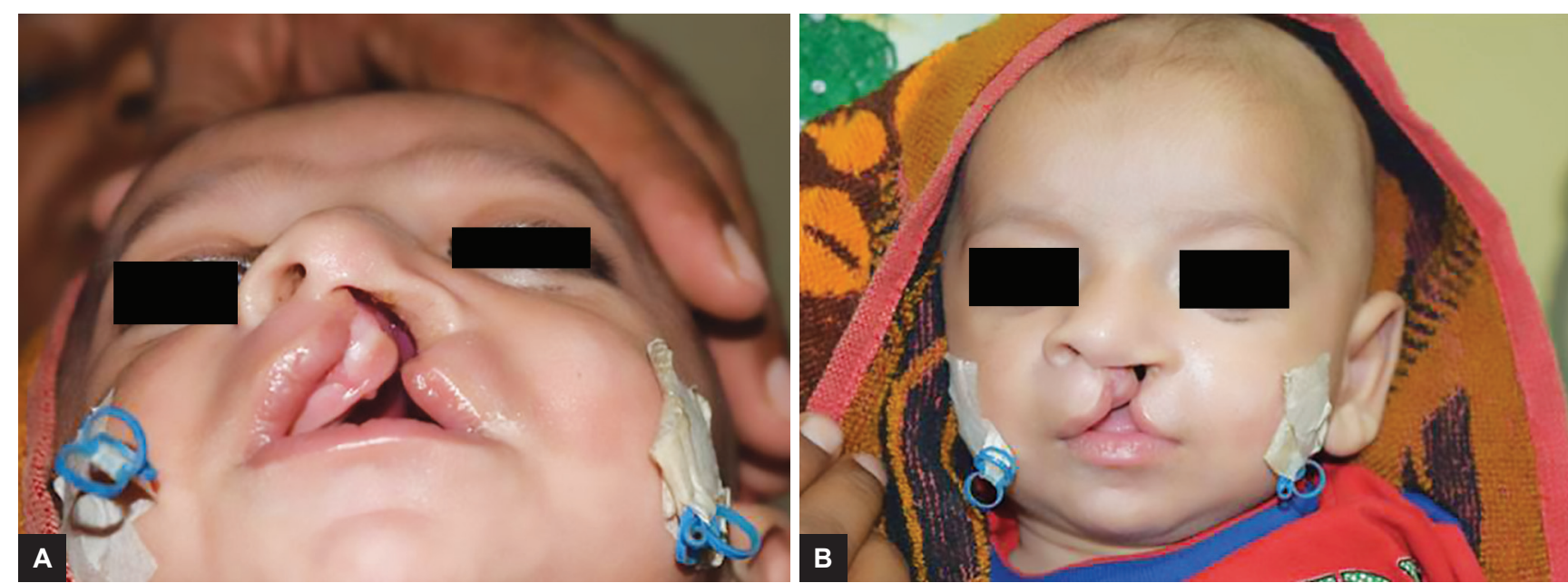

Figs 8 A and B: Postoperative view of the patient showing significant soft tissue closure after PNAM therapy

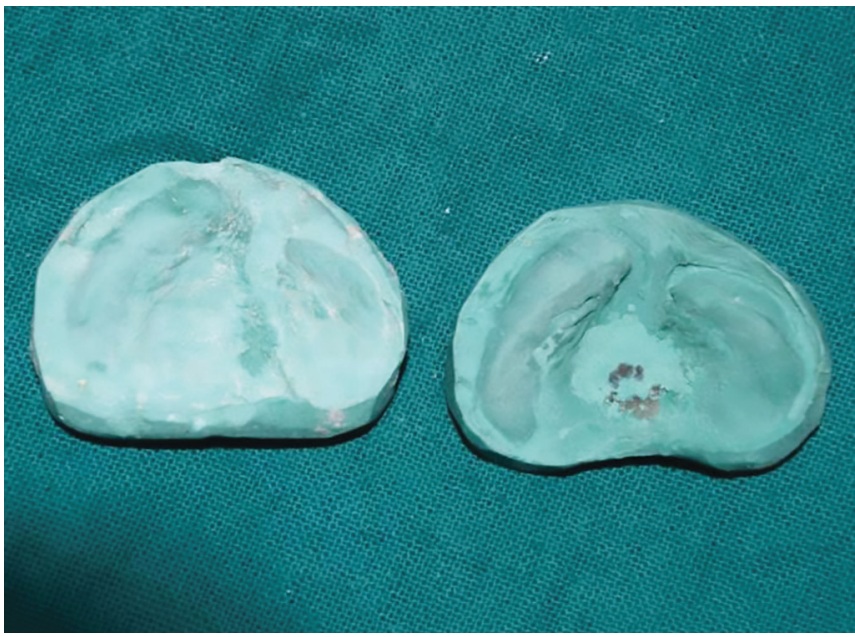

Fig. 9: Comparison between preoperative and postoperative hard tissue closure after PNAM

palate near to fissure) and removing it with pulpy relining matter to apply thrust for resorption, resulting in sealing of cleft gap. The micropore tape and blue elastics were changed to allow continuous retention of the appliance. The parents were guided with detailed directions for lip taping along taping materials and adhesive. The nasal stent was adapted once a week for accurate alar cartilage growth. After PNAM, alveolar molding achieved was satisfactory (Figs 8 and 9). The results of preoperative and postoperative measurements of different variables are shown in Table 1.

\section{DISCUSSION}

The orofacial clefting is the second most common inborn malformation. The facial clefts may involve any portion of face. Most of these craniofacial clefts are unique and are apparently mentioned as Tessier ${ }^{11}$ clefts. The CLCP is especially a treatable deformity with treatment based upon the class and gravity of the cleft.
Table 1: Preoperative and postoperative measurements of extraoral and intraoral variables

\begin{tabular}{lll}
\hline & $\begin{array}{l}\text { Preoperative } \\
(\mathrm{mm})\end{array}$ & $\begin{array}{l}\text { Postoperative } \\
(\mathrm{mm})\end{array}$ \\
\hline Extraoral measurements & & \\
Columella width & 2 & 4 \\
Columella length & 4.5 & 6 \\
Interlip distance & 14 & 5 \\
Intraoral measurements & & \\
Intersegment distance & 11 & 6 \\
Arch width & 38.5 & 42 \\
Greater segment length & 28 & 30 \\
Lesser segment length & 17.5 & 24 \\
\hline
\end{tabular}

The exact etiology still remains unknown. Plenty of genes associated with CLCP have been spotted to contribute the solitary instance of CLCP. This includes in particular order aberration in the genes IRF6, PVRL1, and MSX $1 .{ }^{12}$ The insight relating to genetic entanglement inculpated in growth and differentiation of the midface has been substantially helped by experimentation. The environmental impact may also predispose or intercommunicate with genetics to create orofacial clefting.

The significance of the collective advancement for management of CLCP children cannot be ruled out. The American Cleft Palate Craniofacial Association has published the standard of care in the management of cleft children. The requirements are multifactorial and single independent practitioner approach is frequently harmful for the patient. The children with CLCP are watched by craniofacial team. The craniofacial team consists of an assemblage of experts including an orthodontist, otolaryngologists, pediatric dentist, geneticists, prosthodontists, speech therapists, radiologists, psychologists, feeding specialists, plastic surgeons, and nurses. The family of the patient is always an integral component of the team. ${ }^{11}$

The orthodox method for diagnosis of CLCP is by physical examination during birth. In contrast, the recent 
prenatal diagnosis advances allowed experts to determine orofacial clefts in utero using ultrasonography. ${ }^{13}$ The multidisciplinary management of CLCP deformities should be planned at the proper level encompassing the functional and structural portion encompassed by the deformity. The delivery of care is long-standing. The craniofacial team, in collective effort, determines the treatment procedures. The surgical rehabilitation of fissured lip is usually performed 2 to 3 months after birth. The preferred method is the "rule of 10 " wherein the child should be 10 weeks of age; has minimum of 10 pounds, and has hemoglobin not less than $10 \mathrm{gm}$. The surgical approach for closure in $\mathrm{CP}$ is preferably carried out between 6 and 12 months or it can be covered on an interim basis by a prosthetic device known as obturator.

The early presurgical interventions are usually required to assist tissue approximation while dealing with a wider fissure or anteriorly placed premaxilla before lip repair. The varied procedures include lip taping and adhesion, and/or palatal devices. The prior lip taping gives a gain of "tissue creep" around the cleft as well restricted manipulation of the alveolus and nasal matrix. Furthermore, it does not obstruct the feeding; it is a simple and inexpensive method and is preferably retained up to surgical lip repair. Taping is frequently merged with PNAM for extensive clefts. The movement of the palatal shelves toward each other has a resulting outcome on lip approximation. ${ }^{14}$

Dogliotti et $\mathrm{al}^{15}$ were pioneers for presurgical neonatal nasal remodeling with an infant plate. The PNAM prior to surgery can significantly improve lifelong nasal symmetry among patients with complete unilateral CLCP. In a study, the noteworthy refinement in nasal uniformity has been found in projected length of the alar cartilage of nose, location of super inferior alar groove, position of the mediolateral nasal dome, and nasal bridge deflection. ${ }^{16}$ The logical sense behind the procedure is that surgical repair of lip is revealed more easily, and results in little scar formation that may impede subsequent facial growth.

Grayson and Cutting ${ }^{9}$ reported that the hyaluronic acid levels were found to be increased in the neonatal tissue till about 45 days even after birth. Therefore, the innate molding tendency of neonatal matrix is higher. The PNAM during this phase and preliminary to surgery aids to guide the CLCP components as close as possible and facilitate surgery closure as well as maximize facial esthetics. The subsidiary benefits of PNAM therapy include improvement in feeding, guided advancement of palatal shelves toward approximation, reduction in complex and subsequent revision surgical interventions, improvement of speech, and provides positive psychological impact on the parents, and timely surgical intervention leads to early reduction of deformity.

\section{CONCLUSION}

The children for presurgical orthopedics are registered with serious matter disproportionality. With perfect and well-timed PNAM therapy, the skeletal and soft tissues can be guided into finer arrangement which enables a distinct step repair under most favorable and balanced tissue state with outstanding outcome.

\section{ACKNOWLEDGMENTS}

The authors offer heartfelt gratitude to Dr Samir Dutta, Ex-Senior Professor and Head, Department of Pedodontics and Preventive Dentistry for his continual guidance, enthusiastic approach, and congeniality while performing the procedural facet of the patient care. The case report is dedicated to his hard work and immeasurable knowledge.

\section{REFERENCES}

1. Maull DJ, Grayson BH, Cutting CB, Brecht LL, Brookstein FL, Khorrambadi D, Webb JA, Hurwitz DJ. Long term effects of Nasoalveolar molding on three dimensional nasal shape in unilateral clefts. Cleft palate Craniofac J 1999 Sep;36(5):391-397.

2. Watkins SE, Meyer RE, Strauss RP, Aylsworth AS. Classification, epidemiology, and genetics of orofacial clefts. Clin Plast Surg 2014 Apr;41(2):149-163.

3. Millard DR, Latham RA, Huifen X, Spiro S, Morovic C. Cleft and palate treated by presurgical orthopaedics, GPP and lip adhesion (POPLA) compared with previous lip adhesion method. A preliminary study of serial dental casts. Plast Reconstr Surg 1999 May;103(6):1630-1644.

4. Noordhoff MS. Reconstruction of vermilion in unilateral and bilateral cleft lips. Plast Reconstr Surg 1984 Jan;73(1):52-61.

5. Murthy PS, Deshmukh S, Bhagyalakshmi A, Srilatha K. Pre surgical nasoalveolar molding: changing paradigms in early cleft lip and palate rehabilitation. J Int Oral Health 2013 Apr;5(2):70-80.

6. Patel D, Goyal R, Puri T. Presurgical nasoalveolar moulding an adjunct to facilitate surgical repair in infants with cleft lip and palate. Mod Plast Surg 2013 Jan;3(1):34-42.

7. McNeil CK. Orthodontic procedures in the treatment of congenital cleft palate. Dent Rec (London) 1950 May;70(5): 126-132.

8. Hamrick MW. A chondrol modeling theory-revisited. J Theor Biol 1997 Dec;201(3):201-208

9. Grayson BH, Cutting CB. Presurgical nasoalveolar orthopedic molding in primary correction of the nose, lip, and alveolus of infants born with unilateral and bilateral clefts. Cleft Palate Craniofac J 2001 May;38(3):193-198.

10. Lee, C.; Grayson, BH.; Lin, WY.; Cutting, CB. Long term study of midface growth in unilateral cleft lip and palate patients following gingivoperiosteoplasty. Chapel Hill (NC): American Cleft Palate Craniofacial Association; 1999.

11. Tessier P. Anatomical classification facial, cranio-facial and latero-facial clefts. J Maxillofac Surg 1976 Jun;4(2):69-92.

12. Cox TC. Taking it to the max: the genetic and developmental mechanisms coordinating midfacial morphogenesis and dysmorphology. Clin Genet 2004 Mar;65(3):163-176. 
13. Costello BJ, Edwards SP, Clemens M. Fetal diagnosis and treatment of craniomaxillofacial anomalies. J Oral Maxillofac Surg 2008 Oct;66(10):1985-1995.

14. Nahai FR, Williams JK, Burstein FD, Martin J, Thomas J. The management of cleft lip and palate: pathways for treatment and longitudinal assessment. Semin Plast Surg 2005 Nov;19(4):275-285.
15. Dogliotti PL, Bennun RD, Losoviz E, Ganiewich E. Nonsurgical treatment of nasal deformity in the cleft patient. Rev Ateneo Arg Odont 1991;27:31-35.

16. Barillas I, Dec W, Warren SM, Cutting CB, Grayson BH. Nasoalveolar molding improves long-term nasal symmetry in complete unilateral cleft lip-cleft palate patients. Plast Reconstr Surg 2009 Mar;123(3):1002-1006. 